This is an electronic reprint of the original article. This reprint may differ from the original in pagination and typographic detail.

\author{
Author(s): Heylen, H.; Babcock, C.; Billowes, J.; Bissell, M.L.; Blaum, K.; Campbell, P.; Cheal, B.; \\ Ruiz, R.F. Garcia; Geppert, Ch.; Gins, W.; Kreim, K.; Moore, lain; Neugart, R.; Neyens, \\ G.; Nörtershäuser, W.; Papuga, J.; Yordanov, D.T.
}

Title: $\quad$ Collinear Laser Spectroscopy on Neutron-rich Mn Isotopes Approaching N = 40

Year: $\quad 2015$

Version:

Please cite the original version:

Heylen, H., Babcock, C., Billowes, J., Bissell, M.L., Blaum, K., Campbell, P., Cheal, B., Ruiz, R. G., Geppert, Ch., Gins, W., Kreim, K., Moore, I., Neugart, R., Neyens, G., Nörtershäuser, W., Papuga, J., \& Yordanov, D.T. (2015). Collinear Laser Spectroscopy on Neutron-rich Mn Isotopes Approaching N = 40. Acta Physica Polonica B, 46(3), 699-702. https://doi.org/10.5506/APhysPolB.46.699

All material supplied via JYX is protected by copyright and other intellectual property rights, and duplication or sale of all or part of any of the repository collections is not permitted, except that material may be duplicated by you for your research use or educational purposes in electronic or print form. You must obtain permission for any other use. Electronic or print copies may not be offered, whether for sale or otherwise to anyone who is not an authorised user. 


\title{
COLLINEAR LASER SPECTROSCOPY ON NEUTRON-RICH Mn ISOTOPES APPROACHING $N=40^{*}$
}

\author{
H. Heylen ${ }^{\mathrm{a}}$, C. BabCoCK ${ }^{\mathrm{b}}$, J. Billowes ${ }^{\mathrm{c}}$, M.L. Bissell ${ }^{\mathrm{a}}$, K. Blaum ${ }^{\mathrm{d}}$ \\ P. Campbell ${ }^{\mathrm{c}}$, B. Cheal ${ }^{\mathrm{e}}$, R.F. Garcia Ruiz ${ }^{\mathrm{a}}, \mathrm{CH}$. Geppert ${ }^{\mathrm{f}, \mathrm{g}}$

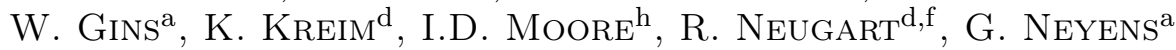 \\ W. NÖRTERShäUSER ${ }^{g}$, J. PAPUGA ${ }^{a}$, D.T. YORDANOV ${ }^{\mathrm{d}}$ \\ ${ }^{a}$ KU Leuven, Instituut voor Kern- en Stralingsfysica, 3001 Leuven, Belgium \\ bISOLDE, CERN, 1211 Geneva 23, Switzerland \\ ${ }^{\mathrm{c}}$ School of Physics and Astronomy, University of Manchester, M13 9PL, UK \\ dMax-Plank-Institut für Kernphysik, 69117 Heidelberg, Germany \\ ${ }^{\text {e}}$ Oliver Lodge Laboratory, Oxford Street, University of Liverpool, L69 7ZE, UK \\ ${ }^{f}$ Johannes Gutenberg-Universität Mainz, Inst. für Kernchemie, 55128, Germany \\ 'Institut für Kernphysik, TU Darmstadt, 64289 Darmstadt, Germany \\ ${ }^{\mathrm{h}}$ Dept. of Physics, University of Jyväskylä, PB 35 (YFL) Jyväskylä, Finland
}

\section{(Received March 23, 2015)}

We have studied ${ }^{51,53-64} \mathrm{Mn}(Z=25)$ via bunched-beam collinear laser spectroscopy at ISOLDE, CERN. Model-independent information on the ground- and isomeric state spins, as well as their $g$-factors is obtained from the measured hyperfine spectra. The spins are essential for further establishing the level schemes in the mass region, while the $g$-factors reveal the changing ground state wave functions in the Mn chain approaching $N=40$.

DOI:10.5506/APhysPolB.46.699

PACS numbers: 21.10.Ky, 21.10.Hw, 27.50.+e, 42.62.Fi

\section{Introduction}

Understanding the structure of nuclei with extreme $N / Z$ ratios is an important challenge in modern nuclear physics research. The neutron-rich $p f$-shell nuclei south of ${ }^{68} \mathrm{Ni}$ are of special interest due to the rapid shell structure evolution for $Z<28$ and $N \approx 40$. A sudden onset of collectivity is observed in the $\mathrm{Fe}(Z=26)[1-3]$ and $\mathrm{Cr}(Z=24)[4,5]$ chains approaching $N=40$, attributed to the occupation of the $\nu g_{9 / 2}$ and $\nu d_{5 / 2}$ intruder orbitals [6]. In the proton-magic Ni chain, the $Z=28$ shell closure has a stabilizing effect such that ${ }^{68} \mathrm{Ni}_{40}$ retains a spherical ground state. However,

* Presented at the Zakopane Conference on Nuclear Physics "Extremes of the Nuclear Landscape", Zakopane, Poland, August 31-September 7, 2014. 
the removal of protons from the $Z=28$ shell results in a reduction of the weak $N=40$ subshell gap under the influence of the proton-neutron interaction. This facilitates the promotion of neutrons across the subshell gap to the $\nu g_{9 / 2}$ and $\nu d_{5 / 2}$ orbitals, accompanied by a large gain in quadrupole correlation energy. For isotopes below $Z=28$, these effects can result in a situation where deformation is energetically favoured for certain $N$ and $Z$ combinations. Although the onset of collectivity is well studied in the neutron-rich $\mathrm{Fe}$ and $\mathrm{Cr}$ nuclei, the experimental information on their oddproton neighbour $\operatorname{Mn}(Z=25)$ is rather limited.

In this work, we report on the measurement of the hyperfine structure of the ${ }^{51,53-64} \mathrm{Mn}$ isotopes $(N=26-39)$ and ${ }^{58 m, 60 m, 62 m} \mathrm{Mn}$ isomers via bunched-beam collinear laser spectroscopy. The hyperfine spectra allow a model-independent extraction of the spins and $g$-factors, observables that give valuable information on the changing shell structure towards $N=40$.

\section{Experimental method}

The bunched-beam collinear laser spectroscopy experiment was performed at the COLLAPs beam line [7] at ISOLDE, CERN. A thick $\mathrm{UC}_{x}$ target was bombarded with a $1.4 \mathrm{GeV}$ proton beam to produce the radioactive $\mathrm{Mn}$ atoms. Following the diffusion out of the hot target, the Mn ions were selectively ionized using the RILIS laser ion source [8]. The ions were accelerated to $40 \mathrm{keV}$ and mass separated in the high resolution separator before they were bunched in the ISCOOL cooler-buncher [9]. The fast ion bunches were then guided to the COLLAPS beam line where a post-acceleration voltage was applied to Doppler-shift the laser frequency across the hyperfine spectrum. After the ions were neutralized by passing through an alkali vapour in the charge exchange cell, the atom bunches were collinearly overlapped with a frequency doubled narrowband dye laser. The laser-induced fluorescence was then detected by up to four PMTs in the light collection region. The continuous background of scattered laser photons was reduced by a factor of $\sim 10^{4}$ by gating the fluorescence signal on the passage of the atom bunch in front of the PMTs. A schematic representation of the setup can be found in [10].

Collinear laser spectroscopy was performed on the ${ }^{6} S_{5 / 2} \rightarrow{ }^{6} P_{3 / 2}$ ground state atomic transition at $289.85 \mathrm{~nm}$. A typical hyperfine spectrum can be found in Fig. 1 (a). The relative peak positions in the spectrum are correlated through the spin and hyperfine parameters $A$ and $B$ [11]. By comparison with the ${ }^{55} \mathrm{Mn}$ reference [12], these parameters give direct information on the $g$-factors and spectroscopic quadrupole moments, $Q_{\mathrm{s}}$, via the relations $g=g_{\text {ref }} A / A_{\text {ref }}$ and $Q_{\mathrm{s}}=Q_{\mathrm{s}, \text { ref }} B / B_{\text {ref }}$. Due to the small quadrupole splitting of the considered atomic transition the precision on the quadrupole moments is very low with relative errors generally larger than $50 \%$. 

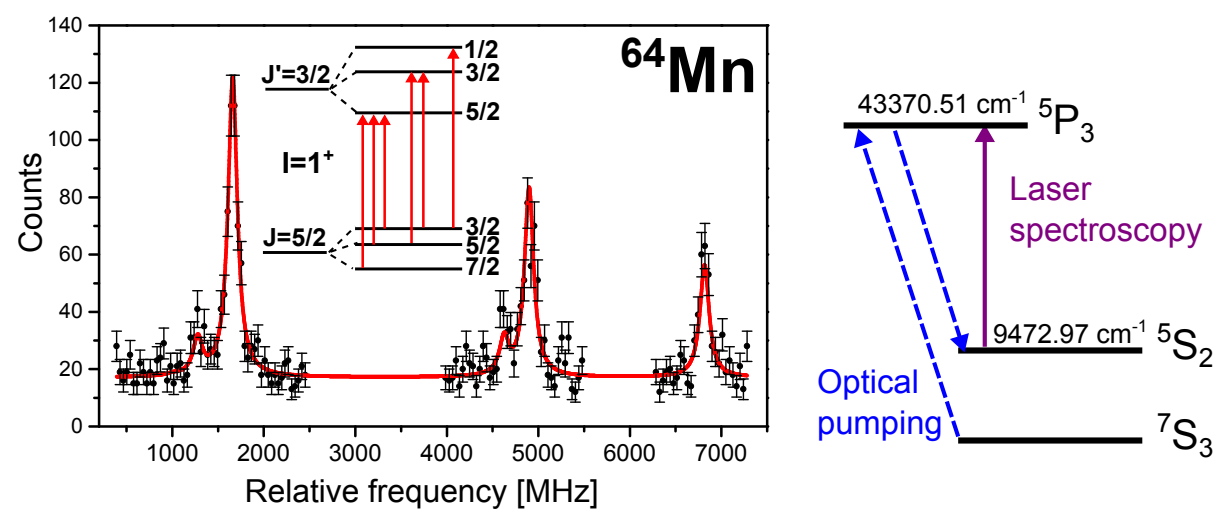

Fig. 1. (a) A sample hyperfine spectrum of ${ }^{64} \mathrm{Mn}$ measured on the neutral atomic ${ }^{6} S_{5 / 2} \rightarrow{ }^{6} P_{3 / 2}$ transition. Each peak in the spectrum corresponds to an allowed optical transition, indicated with the arrows (red) in the hyperfine structure shown as inset. (b) The proposed optical pumping and ionic spectroscopy scheme for future $Q_{\mathrm{s}}$ measurements.

A $\chi^{2}$ minimization procedure was used to analyze the hyperfine spectra. Each peak was fitted with a Lorentzian profile and the relative peak intensities were fixed to the spin-dependent Racah values [13]. A detailed lineshape analysis revealed a small asymmetry which is understood as arising from inelastic processes in the charge exchange cell [14, 15]. Indeed, after neutralization some of the $\mathrm{Mn}$ atoms are excited to the ${ }^{6} P$-triplet at $4.43 \mathrm{eV}$ due to collisions with the alkali-metal vapour. This results in small satellite peaks at the low-energy side of each peak since the excitation energy is taken from the kinetic energy of the fast Mn beam. The addition of one small sidepeak shifted by $4.43 \mathrm{eV}$ with respect to the main peak indeed improved the fit quality, while more sidepeaks did not offer a better reproduction of the experimental peak shape.

\section{Summary and outlook}

We have measured the hyperfine spectra of the ${ }^{51,53-64} \mathrm{Mn}$ ground states as well as of the ${ }^{58 m, 60 m, 62 m} \mathrm{Mn}$ isomers. Spins and $g$-factors were extracted but a precise determination of the quadrupole moment was impossible using the current atomic transition. These results and a detailed discussion will be presented in two forthcoming publications [16, 17].

This work is an important step in the investigation of the shell structure evolution of the Mn chain. Nevertheless, for a deeper understanding of the physics also quadrupole moments are required along with an extension of the data beyond $N=40$. This can only be achieved with an optical transition 
which has a larger quadrupole splitting and which is more efficient. Charlwood and coworkers [18] showed that the $294.92 \mathrm{~nm}$ ionic transition from the ${ }^{5} S_{2}$ metastable state at $9472.97 \mathrm{~cm}^{-1}$ matches these requirements. Laser spectroscopy from this metastable state is intensified by optically pumping [19] the ${ }^{7} S_{3} \rightarrow{ }^{5} P_{3}(230.50 \mathrm{~nm})$ transition in the cooler-buncher. The pumping selectively enhances the population of the metastable state as a result of the strong branching from the ${ }^{5} P_{3}$ to the ${ }^{5} S_{2}$ state, as illustrated in Fig. 1 (b). Due to the extended laser-ion interaction time in the cooler-buncher an efficient pumping process is obtained.

A follow-up collinear laser spectroscopy experiment on Mn using optical pumping in ISCOOL has been accepted at ISOLDE, CERN and will be performed in the near future.

This work was supported by the IAP-project P7/12, the FWO Vlaanderen, GOA 10/010 from KU Leuven, the Max-Planck-Society and the BMBF under contract 05 P12 RDCIC.

\section{REFERENCES}

[1] M. Hannawald et al., Phys. Rev. Lett. 82, 1391 (1999).

[2] J. Ljungvall et al., Phys. Rev. C81, 061301 (2010).

[3] W. Rother et al., Phys. Rev. Lett. 106, 022502 (2011).

[4] H.L. Crawford et al., Phys. Rev. Lett. 110, 242701 (2013).

[5] T. Baugher et al., Phys. Rev. C86, 011305 (2012).

[6] S.M. Lenzi et al., Phys. Rev. C82, 054301 (2010).

[7] R. Neugart, Nucl. Instrum. Methods 186, 165 (1981).

[8] V.N. Fedosseev et al., Rev. Sci. Instrum. 83, 02 A903 (2012).

[9] E. Mané et al., Eur. Phys. J. A42, 503 (2009).

[10] J. Papuga et al., Phys. Rev. C90, 034321 (2014).

[11] B. Cheal, K.T. Flanagan, J. Phys. G: Nucl. Part. Phys. 37, 113101 (2010).

[12] S.J. Davis et al., Phys. Rev. A3, 1220 (1971).

[13] P.C. Magnante, H.H. Stroke, J. Opt. Soc. Am. 59, 836 (1969).

[14] N. Bendali et al., J. Phys. B: At. Mol. Phys. 19, 233 (1986).

[15] K. Kreim et al., Phys. Lett. B731, 97 (2014).

[16] C. Babcock et al., in preparation.

[17] H. Heylen et al., in preparation.

[18] F. Charlwood et al., Phys. Lett. B690, 346 (2010).

[19] B. Cheal et al., Phys. Rev. Lett. 102, 222501 (2009). 